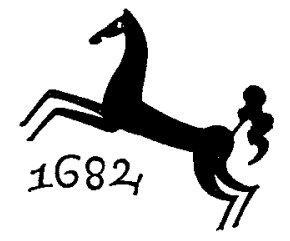

Germanistische Abhandlungen

HOMER IN DER DEUTSCHEN LITERATUR

$$
\text { (I450-I740) }
$$




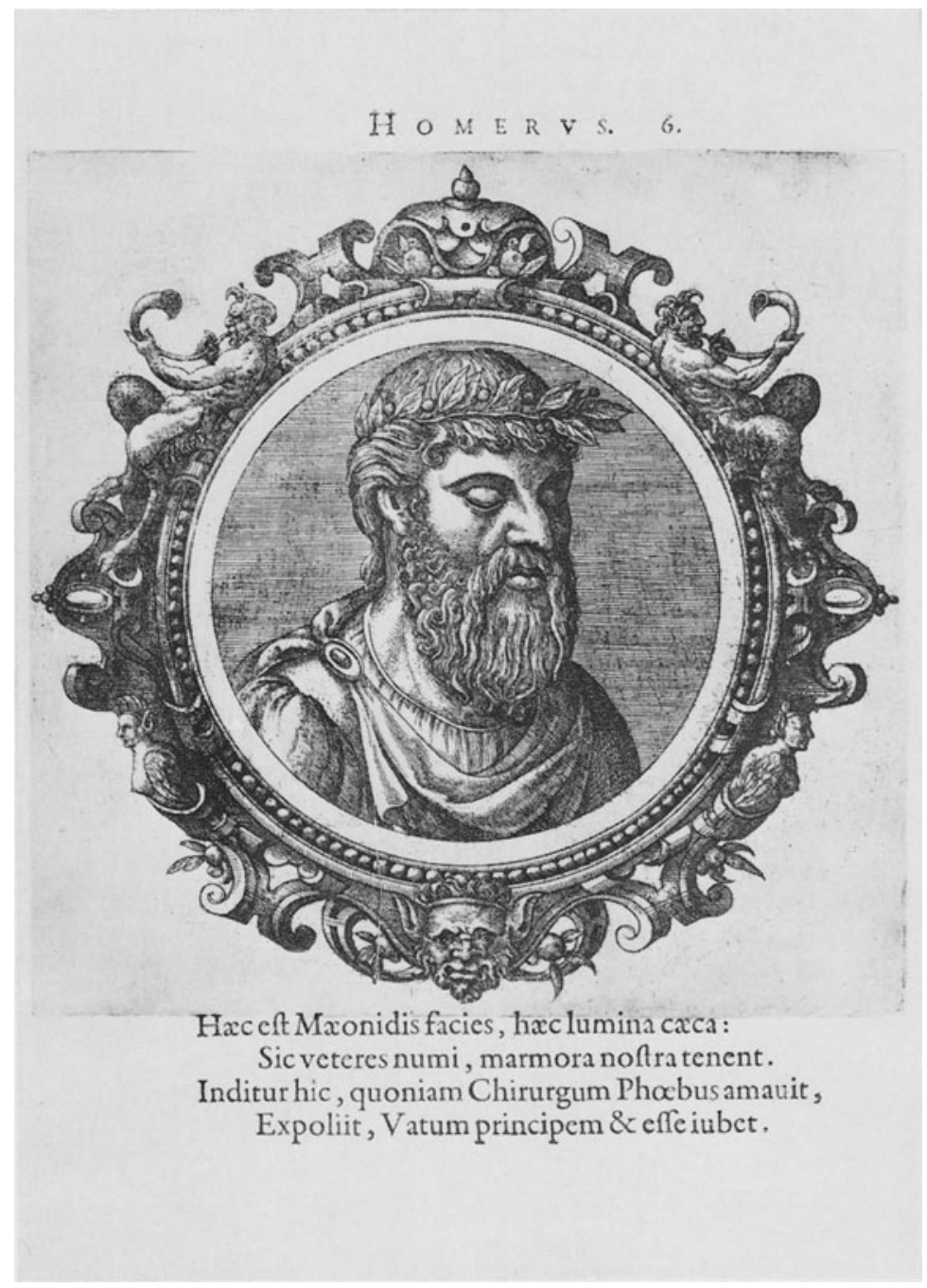

Johannes Sambucus:

ICONES VETERVM ALIQVOT, AC RECENTIVM MEDICORVM, PHILOSOPHORVMQVE, Antwerpen 1574 


\title{
Homer in der deutschen Literatur (I450-I740)
}

\author{
ZUR REZEPTION DER ANTIKE \\ UND ZUR POETOLOGIE DER NEUZEIT
}

MCMLXXII

J. B. METZLERSCHE VERLAGSBUCHHANDLUNG STUTTGART 


\section{GERMANISTISCHE ABHANDLUNGEN 39}

Die Zahlen in [] verweisen auf die Anmerkungen.

ISBN $978-3-476-00249-5$

ISBN 978-3-476-03005-4 (eBook)

DOI 10.1007/978-3-476-03005-4

(C) 1972 Springer-Verlag GmbH Deutschland

Ursprünglich erschienen bei J. B. Metzlersche Verlagsbuchhandlung und Carl Ernst Poeschel Verlag GmbH in Stuttgart 1972 


\section{INHALT}

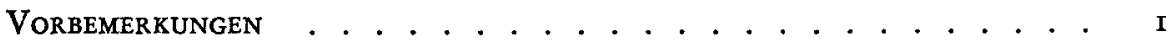

I. KURZER ABRISS DER HOMERISCHEN WIR KUNGSGESCHICHTE

I. Homer-Rezeption im Mittelalter: Von der Verfälschung bis zur Entfremdung ............... . . 4

2. Homer-Rezeption in der italienischen Frïbrenaissance: Sehnsucht nach dem unbekannten Original . . . . . . . . . . . 8

II. Humanistische Homerbewer tungen

I. Homer-Rezeption im Frübhumanismus

a) Traditionelle Fehlperspektiven und Ansätze eines Verstehens . . . II

b) Erste dichtungstheoretische Folgerungen . . . . . . . . . . 22

c) Technische Grundlage und ideelles Gerüst . . . . . . . . . . 30

d) Poetische Apologie und christliche Polemik . . . . . . . . . 34

e) Reuchlins Begriff einer ,poetischen Philosophie . . . . . . . . 40

f) Aktualität und sozialpolitische Zielsetzungen . . . . . . . ${ }_{4}^{46}$

2. Das Homerbild der großen Humanisten

a) Erasmus: der allegorisch-ästhetische Interpretationscirculus . . . s5

b) Vadian: die Einheit aus Abhängigkeit und Vorbildlichkeit . . . . 68

c) Melandhthon: das Rhetorik-Ideal und die Gesetzlichkeit des Humanen . . . . . . . . . . . 72

d) Camerarius: Editionstätigkeit und die Komplexität des Kunstwerkes 86

e) Die Zwingli-Schule: religiös-pädagogische Tendenzliteratur . . . 94 3. Homer-Rezeption im Späthumanismus:

Lateinische Übersetzung und poetische Umwandlung . . . . . . . 98

III. Das Homerbild in der Zeit zwischen Humanismus und Barock

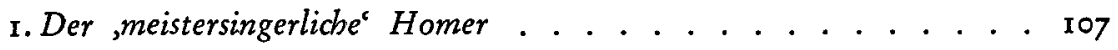

2. Stoffinteresse und Deutungsschematismen ........... $1 \mathrm{I} 7$

EXKurs:

VoRAUSWEISENDE AUSLÄNDISCHE DiCHTUNGSTHEORIEN . . . . . . . I3I

IV. BAROCKE HOMERBEWERTUNGEN

I. Homer-Rezeption im Frübbarock

a) Kritische Uberprüfung und Wertsteigerung . . . . . . . . . $\mathrm{I}_{42}$

b) Epigonale Auslegungen und neue Variationen . . . . . . . 145 
2. Das Homerbild des Opitz und seiner Nacbfolger

a) Das ,poetologische Homermuster . . . . . . . . . . . . . . I49

b) Zwiespalt zwischen Poesie und Religion . . . . . . . . . . . . I57

3. Homers Werk in polybistorischer Sicht . . . . . . . . . . . . . 166

a) Homer in verschiedenartigen Sammelwerken . . . . . . . . . 166

b) Werke über Homer . . . . . . . . . . . . . . . . . . . . 169

V. HomerbeWERTUNG IN SPÄtbarock UND AUfKLÄrung

I. Die Odyssee als beroischer Roman . . . . . . . . . . . . . . . 178

2. Dramatisierung und Eposerneuerung . . . . . . . . . . . . . . I86

3. Der Widerstreit in den, rationalistischen" Stellungnabmen . . . . . 194

4. Abschluß und Neuansatz: das Gesetz und das Wunderbare . . . . . 204

Ausblick . . . . . . . . . . . . . . . . . . . 209

ANHANG:

ANMERKUNGEN . . . . . . . . . . . . . . . . . . . . . . . . 213

LITERATURVERZEICHNIS . . . . . . . . . . . . . . . . . . . . 280

PeRSONENREgISTER . . . . . . . . . . . . . . . . . . . . 307 


\section{AbKürzungen}

$A D B$

de BackerSommervogel

Capelli

$D W B$

Ellinger

EHS

$G d T$

Goedeke

Orbis latinus

Graesse

Holtzmann

Hunger

Jöcher

$K L L$

Lesky

$L A W$

$L T h K$

Manitius

Nadler
Allgemeine deutsche Biographie, Leipzig $x 875 \mathrm{ff}$.

Bibliothèque de la Compagnie de Jésus ... par les Pères Augustin et Aloys de Backer et par Carlos Sommervogel, Brüssel/Paris I $890 \mathrm{ff}$.

Adriano Capelli: Lexicon Abbreviaturum, Mailand 1899.

Jacob und Wilhelm Grimm: Deutsches Wörterbuch, Leipzig I8 $54 \mathrm{ff}$.

Georg Ellinger: Geschichte der neulateinischen Literatur Deutschlands im 16. Jahrhundert, Berlin/Leipzig $1929 \mathrm{ff}$.

Emblemata. Handbuch zur Sinnbildkunst des XVI. und XVII. Jh., hg. von Arthur Henkel und Albrecht Schöne im Auftrage der Göttinger Akademie der Wissenschaften, Stuttgart 1967.

Geschichte der Textüberlieferung. Bd. I: Uberlieferungsgeschichte der antiken Literatur, Zürich 196I.

Grundriß zur Geschichte der deutschen Dichtung. Von Karl Goedeke, ${ }^{2}$ Dresden $188_{4} \mathrm{ff}$.

Johann Georg Theodor Graesse: Orbis latinus oder Verzeichnis der lateinischen Benennungen (Neudrudk der ersten Auflage Dresden r86r), Amsterdam 1969.

Johann Georg Theodor Graesse: Trésor de livres rares et précieux, Dresden $1859 \mathrm{ff}$.

Holtzmann, M., und H. Bohatta: Deutsches Pseudonymen-Lexikon, Wien 1906.

Herbert Hunger: Lexikon der griechischen und römischen Mythologie (mit Hinweisen auf das Fortwirken antiker Stoffe und Motive), ${ }^{5}$ Wien 1959.

Allgemeines Gelehrten=LEXICON, hg. von Christian Gottlieb Jöcher, Leipzig 1750 ff.

Kindlers Literatur Lexikon, begründet von Wolfgang von Einsiedel unter Mitarbeit zahlreicher Fachberater, Zürich 1970 ff.

Albin Lesky: Geschichte der griechischen Literatur, ${ }^{2}$ Bern/München 1963.

Lexikon der Alten Welt. Redaktion Klaus Bartels und Ludwig Huber, Zürich/Stuttgart 1965.

Lexikon für Theologie und Kirche, begr. von Michael Buchberger, hg. von Josef Höfer und Karl Rahner, ${ }^{2}$ Freiburg $1957 \mathrm{ff}$.

Max Manitius: Geschichte der lateinischen Literatur des Mittelalters, München Igr Iff.

Literaturgeschichte der deutschen Stämme und Landschaften. Von Josef Nadler, ${ }^{3}$ Regensburg I $929 \mathrm{ff}$. 
$N d B$

Newald

$R A C$

$R E$

$R G G$

$R L$

Roscher

Tusculum

Voigt

Wilpert

Zedler
Neue deutsche Biographie. Hg. von der Historischen Kommission bei der Bayerischen Akademie der Wissenschaften, Berlin $1953 \mathrm{ff}$.

Die deutsche Literatur vom Späthumanismus zur Empfindsamkeit (I 570-1750). Von Richard Newald, Bd. 5 der Geschichte der deutschen Literatur, ${ }^{4}$ München 1963 .

Reallexikon für Antike und Christentum. Sachwörterbuch zur Auseinandersetzung des Christentums mit der antiken Welt, hg. von Theodor Klauser, Stuttgart $1950 \mathrm{ff}$.

Paulys Real=Encyclopädie der Classischen Altertumswissenschaft. Neue Bearbeitung, begr. von Georg Wissowa, fortgeführt von Wilhelm Kroll und Karl Mittelhaus, Stuttgart i $894 \mathrm{ff}$.

Die Religion in Geschichte und Gegenwart. Handwörterbuch für Theologie und Religionswissenschaft, hg. von Kurt Golling, ${ }^{3}$ Tübingen r 957 ff.

Reallexikon der deutschen Literaturgeschichte. Begr. von Paul Merker und Wolfgang Stammler, neu bearbeitet und hg. von Werner Kohlschmidt und Wolfgang Mohr, Berlin $1958 \mathrm{ff}$.

W. H. Roscher (Hrsg.): Ausführliches Lexikon der griechischen und römischen Mythologie, Leipzig I $884 \mathrm{ff}$.

Tusculum-Lexikon griechischer und lateinischer Autoren des Altertums und des Mittelalters. Völlig neu bearbeitet von W. Buchwald, A. Hohlweg und O. Prinz, München 1963 .

Georg Voigt: Die Wiederbelebung des classischen Alterthums oder das erste Jh. des Humanismus, Berlin $1880 \mathrm{f}$.

Lexikon der Weltliteratur. Biographisch-bibliographisches Handwörterbuch nach Autoren und anonymen Werken. Unter Mitarbeit zahlreicher Fachgelehrter hg. von Gero von Wilpert, Stuttgart 1963 .

Johann Heinrich Zedler: Grosses vollständiges UNIVERSAL LEXICON Aller Wissenschafften und Künste, Halle/Leipzig $1732 \mathrm{ff}$. 


\title{
VORBEMERKUNGEN
}

\begin{abstract}
"Aber das Leben ist vorgewirkt in den Alten, erkannt in den Alten und gesehen in den Alten *

Niklas von Wyle [r]

"Homer ist also ... folglich ein recht großer Geist, ein Mann, von besonderer Fähigkeit gewesen ... Viele haben ihn ohne Einsicht gepriesen, damit sie nur dafür angesehen würden, als ob sie ihn verstanden hätten: viele haben ihn auch ohne Grund getadelt, damit sie nur das Ansehen hätten, als verstünden sie besser, was zur Poesie gehört, als andere, die den Homer vertheidigten und lobten« Johann Christoph Gottsched [2]
\end{abstract}

Úberraschend klingen die Worte, die Niklas von Wyle schon am Anfang einer Auseinandersetzung mit der antiken Literatur in Deutschland ausspricht. In ihnen erscheint jedoch die Möglichkeit eines geistigen Nacherlebens mehr erahnt als erfahren. Sie bedeuten nicht Ausdruck eines eigenen >Bekenntnisses‘; sie stellen das Programm auf, das für die folgende Zeit gültig werden sollte. Dessen Inhalt wird entscheidend durch die Begegnung mit Homer, dem wirkungsstärksten Dichter des griechischen Kulturbereiches, geprägt. Die Beurteilung seiner Person und seines Werkes ist ebenso vielseitig wie unterschiedlich. Von I500 an häufen sich die Aussagen über diesen Autor und erhalten oft ein starkes Gewicht durch die Absicht, die mit ihnen verbunden wird: In der Außerung über Homer wird zugleich auch eine Antwort auf die Frage nach Wert und Nutzen der Poesie im allgemeinen und Sinn und Stellung der profanen Literatur im besonderen gesucht.

Am Ende dieses ersten lang andauernden Zeitabschnitts, in dem Homers Werk fester Bestandteil des deutschen Kulturlebens geworden ist, kritisiert Gottsched zahlreiche, darunter sicherlich auch deutsche Schriftsteller, die sich zu Homer in irgendeine Beziehung gesetzt haben. Die zwei Extreme: das Lob der einen, die in Homer den vollkommenen Dichter erblicken, der Tadel der anderen, die die homerische Epik als veraltetes und fehlerhaftes >Modell abtun. Gottscheds Haltung mutet uns wie ein Rückblick auf eine vergangene Epoche an, der nun in einer neuen Zeit eine neue Homerbetrachtung folgen sollte. In der zweiten Hälfte des $\mathrm{r} 8$. Jh. setzt dann ein stark gefühlsbetontes Nachempfinden der homerischen Didhtung ein. So sehr dadurch Einsichten in die Eigenart der Ilias und der Odyssee gewonnen sein mögen - sie entsprechen nicht dem, was Gottsched gewollt, wenn auch selbst nicht erreicht hat: eine >kritische $<$ Ubberprüfung des alten humanistisch-barocken Homerbildes. 
Eine Arbeit, die die Erfüllung dieser Forderung heute nachholen möchte, muß jedoch früher ansetzen. Personen und Ansichten der vorklassischen Epochen sind uns nur noch vereinzelt bekannt, da die Goethezeit mit ihrer Deutung Homers als eines Originalgenies uns den Blick für die vielen Vorgänger verstellt hat. Unsere besondere Aufgabe wird es daher sein, auf die Quellen selbst zurückzugreifen und die in ihnen entwickelten Ansatzpunkte und Maßstäbe einer Homerbewertung aufzudecken. Wir folgen damit zwar dem humanistischen Aufruf »Ad fontes«, verkennen aber nicht die Schwierigkeit unserer Ausgangsposition: Von der Gegenwart aus beurteilen wir eine längst vergangene Zeit, die sich wiederum mit dem ältesten Autor der Antike beschäftigt hat. Gerade diese Verdoppelung der Reflexion zeigt sehr deutlich, in welchem Maße der eigene Standort von der augenblicklichen historischen Konstellation abhängig und daher überholbar ist. Um nun die Voraussetzungen unseres Standortes zu umreißen, sollen in den folgenden Vorbemerkungen einige Richtlinien angegeben werden, die Aufbau und Darstellung dieser Arbeit bestimmt haben.

I. Unter »Homer wird, entsprechend den Auffassungen in den behandelten Epochen, ein einziger Autor verstanden, der Ilias und Odyssee, oft aber auch Batrachomyomachie und Hymnen gedichtet hat (abweichende Einzelfälle werden gesondert angezeigt).

2. Bei der Wertung der herangezogenen Quellen mußten natürlich vorrangig die historischen Bedingungen berüdksichtigt werden. Stets erkennbar sein sollte jedoch auch das (keineswegs eigenständige) Homerbild des Verfassers, der sich zu einer ssynthetischen', gleichermaßen traditionelle Bindung und originale Leistung beachtenden Deutung bekennt.

3. Literarisches Selbstverständnis und Deutung vergangener Literaturen korrespondieren in dem behandelten Zeitabschnitt nicht nur, sondern bieten sich meistens sogar als identische Aussagen an, weil - ohne historisches Einschätzungsvermögen die eigene Vorstellung in den vorliegenden Text hineinprojiziert wird. Daher wird bei jeder >Rezeption der Antiker zugleich auch eine (frühneuzeitliche) >Poetologie mitentworfen.

4. Der Terminus "Deutsche Literatur « wäre irreführend, wenn man darunter eine deutschsprachige Literatur verstände. Überwiegend mußten neulateinische Schriften beachtet werden, sogar noch im Barock, da wissenschaftliche Texte fast immer lateinisch verfaßt wurden.

5. Die zitierten Stellen stammen meist aus philologischen Abhandlungen und dichtungstheoretischen Auslegungen. Daß dabei auch Zeugnisse höchst unterschiedlicher Art nebeneinander gestellt, wenn auch nicht einheitlich beurteilt wurden, konnte wegen der angestrebten Vollständigkeit nicht vermieden werden. - Zwischen Poetiken und werkimmanenten Aussagen über >Poesier sollte nur in seltenen Fällen unterschieden werden, da lediglich nach der Außerung über Homer gefragt werden mußte. Auch der Kontext konnte nur dann Beachtung finden, wenn er diese Äußerung beeinflußte.

6. Die Gewichtverteilung bei der Behandlung der verschiedenen Epochen ist ungleichmäßig. Einer ausführlichen Berücksichtigung des 2 Humanismus` (1450-1600) 
folgen kürzere Kapitel über >Barock < und vor allem über >Aufklärung < $(1600-1740)$. Wesentlich war die weitaus größere Materialfülle aus der humanistischen Epoche. Außerdem mußten besonders die Anfänge deutlich herausgearbeitet werden; in den nachfolgenden Zeitabschnitten sollten hauptsächlich neuere Sichtweisen hervorgehoben werden.

7. Um der jeweiligen Gesamtansicht gerecht zu werden, ließen sich Wiederholungen nicht immer vermeiden. So berühren sich gerade die großen Persönlichkeiten in vielen Punkten, und ihre Abweichungen voneinander heben sich erst deutlich ab, wenn der gemeinsame Hintergrund aufgedeckt ist. Wiederholungen, v. a. im zweiten T'eil, mußten zudem beibehalten werden, da der Kompendium-Charakter der Arbeit gewahrt bleiben sollte.

8. Material und Darstellung bedingten einander. Denn zuerst einmal mußten die Quellen angeführt werden, dann erst konnte deren Stellenwert innerhalb ähnlicher oder verschiedener Texte herausgearbeitet werden. Eine (klarere) Komposition nach übergeordneten Gesichtspunkten verbot sich also wegen der primär erforderlichen Belegsammlung, und eine mögliche zusätzliche Systematisierung hätte die an sich schon umfangreiche Arbeit zu sehr anschwellen lassen.

9. Einen geschlossenen Forschungsbericht zu bieten, war nicht möglich, da selbst Georg Finsler in seinem verdienstvollen Buch über das Nachleben Homers nur wenige Beispiele aus dem deutschen Humanismus angeführt und das Barockzeitalter sogar völlig übergangen hatte. Die thematisch oft eng begrenzten Stellungnahmen aus der Sekundärliteratur wurden deshalb an der jeweiligen Stelle im Text oder in den Anmerkungen berücksichtigt. Dabei überraschte zweierlei: Unsere Fragestellung wurde meist lediglich in Form einer Randbemerkung berührt, und zur Deutung der Quellen konnten wir nur selten neuere wissenschaftliche Abhandlungen heranziehen.

Io. Aufgabe unserer Untersuchung war es, die verschiedenen Phasen einer Erweiterung und Anreicherung des Homerbildes zu analysieren. Trotz deutlicher Entwicklungsstufen und scharfer Gegensätze in der Beurteilung sollte die Zusammengehörigkeit der behandelten Epochen betont werden, um deren Homerbild als einheitlichen Entwurf gegen die nachfolgende andersgeartete Bewertung abzugrenzen. 